\title{
Avaliação radiográfica e tomográfica dos seios nasais de gatos com doenças sinonasais crônicas
}

\author{
[Radiographic and tomographic evaluation of nasal sinus in ten cats with chronic sinonasal diseases] \\ R. Zanatta e J.C. Canola \\ Faculdade de Ciências Agrárias e Veterinárias - UNESP \\ Via de Acesso Paulo Donato Castellane, s/n. \\ 14883-800 - Jaboticabal, SP
}

\begin{abstract}
RESUMO
Avaliaram-se os seios nasais de 10 gatos mestiços, adultos, acometidos por afecções sinonasais, por meio de exames radiográfico e tomográfico, e descreveram-se as vantagens e desvantagens dos métodos. $\mathrm{O}$ grande diferencial do exame de tomografia computadorizada em relação ao exame radiográfico, na avaliação dos seios nasais, foi a detecção de alterações nos seios esfenoides e a maior facilidade em localizar a lateralidade e o grau de envolvimento de alterações dos seios frontais.
\end{abstract}

Palavras-chave: gato, radiografia, tomografia computadorizada, doenças sinonasais

\begin{abstract}
The purpose of this study was to evaluate the nasal sinus of ten adult mixed-breed cats with sinonasal diseases, using radiography and computed tomography to describe the advantages and disadvantages of the methods. In the evaluation of the nasal sinus, the advantages of the CT examination regarding the radiography had been the detection of alterations in the sphenoid sinus and the easiness in locating the side affected and the degree of involvement of the frontal sinus.
\end{abstract}

Keywords: cats, radiography, computed tomography, sinonasal diseases

\section{INTRODUÇÃO}

Os seios nasais são espaços preenchidos por ar, recobertos por mucosa, os quais se comunicam com a cavidade nasal. O sistema de seios do gato compreende compartimentos frontal e esfenoide, dentre os quais o frontal é o mais importante (Dyce et al., 2004). No gato, os seios frontais não são divididos, ao contrário do que ocorre no cão, e ocupam a parte dorsal da cabeça entre a cavidade nasal, a cavidade craniana e as órbitas. Os seios esfenoides são um espaço, relativamente pequeno, na junção das asas e do corpo do osso pré-esfenoide. No gato, contêm parte do quarto osso endoturbinado.

A função dos seios nasais é obscura. Acredita-se que eles conferem certa proteção térmica e mecânica às cavidades orbitária, nasal e craniana, aumentam as áreas do crânio disponíveis para inserção muscular, sem elevar indevidamente o peso, e influenciam na ressonância da voz. Todavia, se os seios têm função questionável no animal normal, nas enfermidades são clinicamente importantes (Norris e Laing, 1985). Os problemas primários dos seios nasais são de rara ocorrência. $\mathrm{O}$ envolvimento dos seios frontais nas afecções nasais deve-se geralmente à sua drenagem deficiente por meio de conchas etmoidais enfermas, ou à extensão de neoplasia da cavidade nasal (Birchard, 1995; Haagen, 2005).

A rinossinusite crônica idiopática e a neoplasia nasal são as causas mais comuns de doenças sinonasais crônicas em gatos. Pólipos

Recebido em 21 de maio de 2010

Aceito em 13 de abril de 2011

E-mail: rosana.zanatta@gmail.com 
nasofaríngeos, rinite fúngica, corpos estranhos nasais e doenças dentais são causas menos frequentes de rinite crônica em gatos (Henderson et al., 2004; Kuehn, 2006; Malinowski, 2006). Os sinais clínicos mais frequentes são espirro, corrimento nasal e estertor respiratório (Michiels et al., 2003).

Dentre os métodos de diagnóstico por imagem, a radiografia é amplamente utilizada na avaliação de animais com sinais clínicos de doenças sinonasais, por ser um método acessível e não invasivo. A qualidade das radiografias de estruturas faciais é altamente dependente do correto posicionamento do paciente, daí a necessidade do uso de anestesia. O estudo radiográfico da cabeça é dificultado pela disposição anatômica dos ossos da região, resultando em imagem com sobreposição de estruturas (Park et al., 1992). Como o seio frontal é pequeno nos gatos, algumas lesões podem não ser detectadas radiograficamente (Schoenborn et al., 2003). Os seios frontais são melhor visibilizados nas projeções radiográficas lateral e frontal da cabeça. Na lateral, eles são claramente observados, sobrepostos um ao outro e sobre a órbita, com opacidade aérea e septos ósseos finos em seu interior. Na projeção frontal (rostrocaudal), uma fração de cada seio é visibilizada rostralmente à cabeça. Os seios etmoidais são de difícil visibilização nas radiografias (Kealy e McAllister, 2005). As doenças sinonasais resultam em achados radiográficos que variam desde discretos aumentos de radiopacidade até lesões líticas e presença de massas expansivas (Pownder et al., 2006).

O surgimento da tomografia computadorizada (TC) mudou o modo como a anatomia do paciente é observada, por permitir visibilizar imagens em planos, eliminando as sobreposições anatômicas, além de determinar a extensão da lesão. A anatomia tomográfica dos seios nasais sem alterações já foi descrita (Losonsky et al., 1997). Em geral, a TC fornece melhor localização das lesões e determina a extensão da doença nos seios nasais, em relação à radiografia. Por essa capacidade, a TC auxilia na escolha do tratamento e para a realização de biopsias ou rinoscopias (Johnson e Wisner, 2007). Os achados frequentemente observados na TC de gatos com doença sinonasal incluem opacificação da cavidade nasal e seios nasais, lise dos ossos nasal e frontal e destruição dos turbinados nasais (Tromblee et al., 2006; Karnik et al., 2009; Scrivani, 2009).

Este estudo teve os objetivos de avaliar os seios nasais de gatos mestiços, adultos, acometidos por afecções sinonasais crônicas, por meio de exames radiográfico e tomográfico, e descrever as vantagens de cada método, com base nos resultados obtidos.

\section{MATERIAL E MÉTODOS}

Os critérios de inclusão dos animais utilizados foram a presença de um ou mais sinais clínicos de doenças sinonasais, como espirro, corrimento nasal e ocular, estertor respiratório e/ou deformidade facial em gatos adultos, provenientes do gatil do Laboratório de Pesquisa em Nutrição e Doenças Nutricionais de Cães e Gatos, mantidos em gaiolas individuais no período noturno e soltos em ambiente coletivo durante o dia para a prática de atividade física voluntária. Ainda, os animais deveriam apresentar histórico de doença sinonasal recorrente e ter sido tratados para tal afecção uma ou mais vezes. Dez gatos, seis fêmeas e quatro machos, com idade desconhecida, todos adultos e sem raça definida, preencheram esses critérios.

Antes da realização dos exames radiográficos e tomográficos, sob jejum alimentar de 12 horas, os animais foram anestesiados com cloridrato de levomepromazina (Neozine $5 \mathrm{mg} / \mathrm{mL}$ - Aventis Pharma LTDA. - 0800 168040), tiletamina e zolazepam (Zoletil 50mg/mL - Virbac do Brasil Indústria e Comércio LTDA. - São Paulo - 0800 136533) nas doses respectivas de $0,5,2,5$ e $2,5 \mathrm{mg} / \mathrm{kg}$ de peso corporal, administradas por via intramuscular em dose única. Distribuíram-se os 10 gatos em dois grupos de igual número, e os exames de cada grupo foram realizados em dias diferentes. Obtiveram-se radiografias da cabeça e, em seguida, imagens tomográficas. Os exames radiográficos foram realizados com aparelho Siemens, modelo Tridoros 812E (Siemens Medical Solutions USA, Inc. - 51 Valley Stream Parkway Malvern, PA 19355), com capacidade para $800 \mathrm{~mA}$. Obtiveram-se radiografias da cabeça em projeções lateral direita e dorsoventral, com $38 \mathrm{kVp}$ e $40 \mathrm{kVp}$, respectivamente, $4 \mathrm{mAs}$ e $400 \mathrm{~mA}$. 
Os exames de TC foram realizados utilizando-se um aparelho Philips, modelo Tomoscan LX/C (Philips Medical Systems - 1550 Sawgrass Corporate Parkway Sunrise, FL 33323 USA). As imagens foram obtidas com o animal em decúbito esternal, com os membros torácicos estendidos cranialmente e os pélvicos caudalmente. Os planos transversais seriados da cabeça tiveram intervalos de $3 \mathrm{~mm}$, iniciando-se na extremidade rostral da cabeça até a articulação atlanto-occipital. As imagens obtidas com 80 ou $120 \mathrm{kVp}$ e $75 \mathrm{~mA}$, com janela para tecido ósseo, foram impressas em cópias rígidas; não foi utilizado contraste iodado.

As imagens radiográficas e tomográficas dos seios nasais foram avaliadas segundo os seguintes critérios: localização da alteração (frontal direito/esquerdo, nenhum; esfenoide direito/esquerdo, nenhum), grau da alteração (completo, incompleto, nenhum), alterações na opacidade (aumentada, reduzida, ausente), presença de massas e alterações dos ossos que delimitam os seios nasais (lise, desvio ou proliferação), presença de desvio dos septos dos seios frontais e esfenoides. Além disso, foi avaliado o envolvimento de estruturas que poderiam estar acometidas com doenças sinonasais, como cavidade nasal, septo nasal, nasofaringe, cavidade timpânica, ossos adjacentes e dentes. A facilidade na realização, a segurança para paciente e pessoal envolvido e a disponibilidade dos exames também foram observadas durante o estudo.

\section{RESULTADOS E DISCUSSÃO}

$\mathrm{Na}$ avaliação radiográfica, observaram-se alterações na opacidade dos seios frontais (Fig. 1) em $90 \%$ dos gatos. Apesar da amostra pequena, apenas 10 gatos, esses achados corroboram com a afirmação de O'Brien et al. (1996) de que a opacificação dos seios frontais é uma das alterações mais frequentes em gatos com doenças sinonasais, bem como a opacificação da cavidade nasal. Entre as imagens dos seios frontais com alteração, $66,7 \%$ eram lesões bilaterais e $22,2 \%$ unilaterais (seio frontal direito), e em um gato a opacificação foi observada em projeção lateral. Nesta última, não foi possível definir a lateralidade da lesão na projeção dorsoventral, devido ao posicionamento discretamente oblíquo, que levou à sobreposição do ramo horizontal da mandíbula sobre o seio frontal direito. $O$ grau de alteração foi incompleto em todos os gatos, isto é, o aumento de opacidade não envolvia o seio frontal na sua totalidade. Este dado foi de difícil avaliação, pois parte do seio frontal ficou sobreposto pelo ramo horizontal da mandíbula. Neste trabalho, não foi realizada a projeção frontal, que poderia ter auxiliado a classificação das lesões quanto à lateralidade e ao grau de envolvimento, lembrando que, mesmo nessa projeção, apenas parte dos seios frontais pode ser visibilizada.

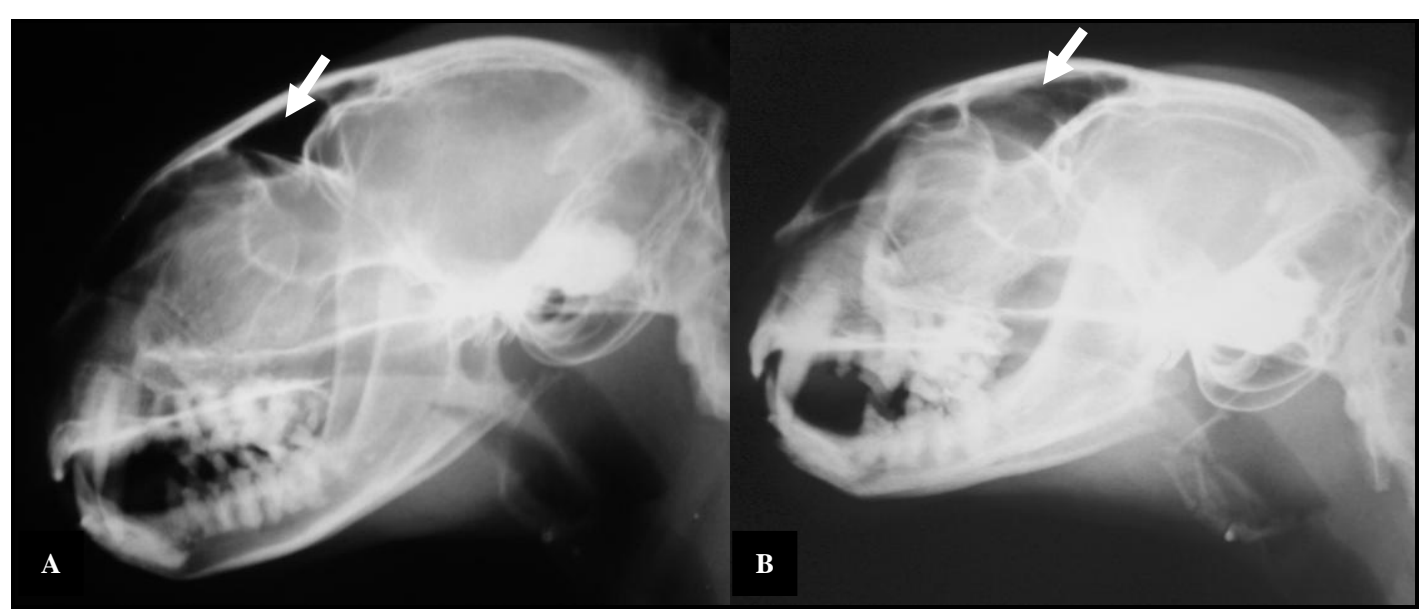

Figura 1. Imagens de radiografias da cabeça, em projeção laterolateral, de gatos sem raça definida, com sinais da doença sinonasal. A: Seio frontal sem alterações (seta). B: Aumento de opacidade do seio frontal (seta). 
Não foram detectadas alterações nos seios esfenoides e nos ossos que delimitam os seios nasais, nem a presença de massas e/ou desvio do septo nas radiografias. Quanto ao envolvimento de outras estruturas geralmente envolvidas nos quadros de doenças sinonasais, foram visibilizados aumento de opacidade da cavidade nasal em sete gatos, aumento de opacidade da cavidade timpânica em três gatos e perda de um ou mais dentes incisivos superiores e/ou inferiores em três.
$\mathrm{Na}$ avaliação tomográfica dos seios nasais, foram detectadas alterações de opacidade (aumento) dos seios frontais $(100 \%)$ e dos seios esfenoides (60\%) (Fig. 2 e 3). Nenhuma alteração nos ossos que delimitam os seios nasais foi observada. Também não foi detectado desvio de septo dos seios nasais, que é raro segundo Reetz et al. (2006). Quanto à lateralidade e ao grau de envolvimento, as alterações nos seios nasais (frontais e esfenoides) foram $100 \%$ bilaterais e incompletas.

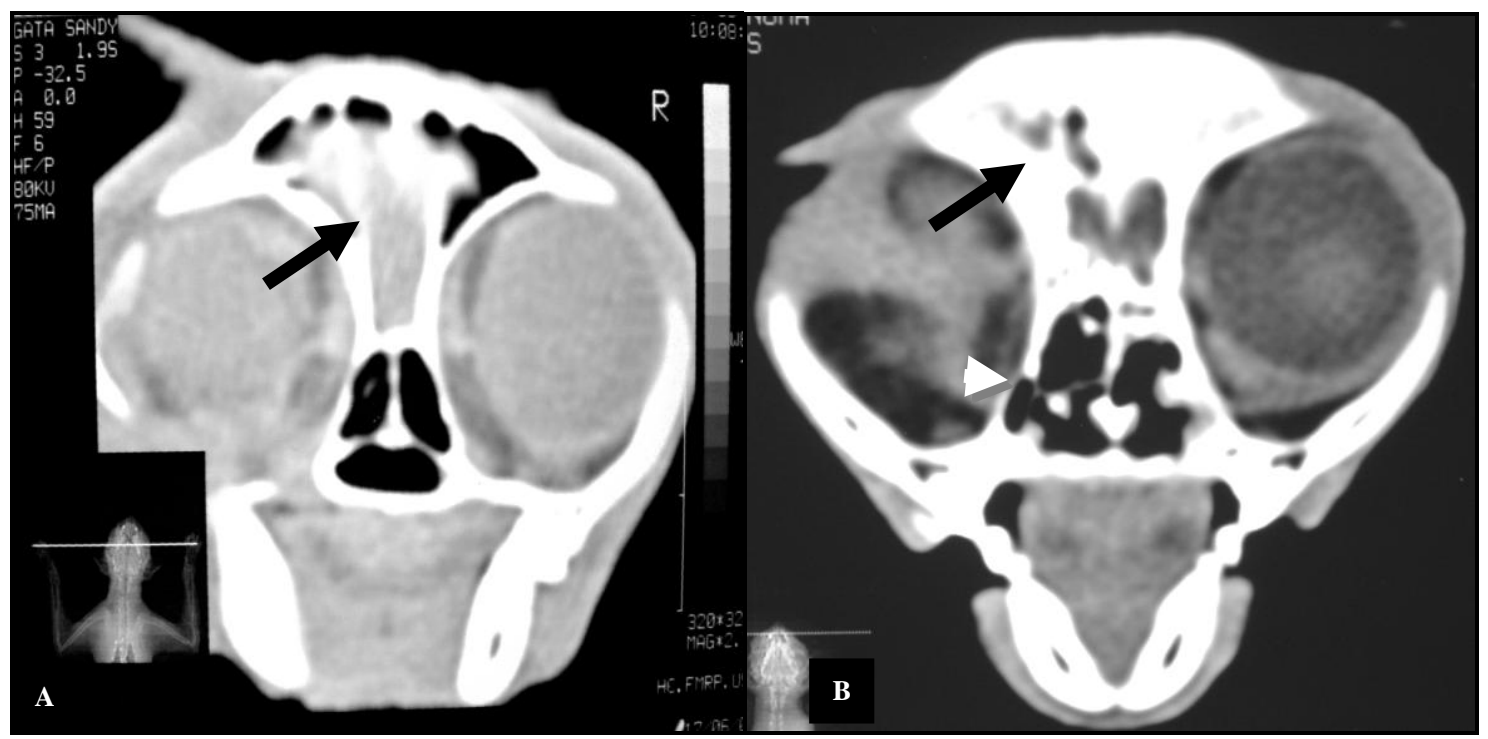

Figura 2. Imagem de tomografia computadorizada em corte transversal, utilizando janela para tecido ósseo, da cabeça de gatos, sem raça definida, com sinais clínicos de doença sinonasal. A e B: Radiodensidade aumentada envolvendo parcialmente os seios frontais direito e esquerdo (setas). Lise do osso lacrimal (ponta de seta).

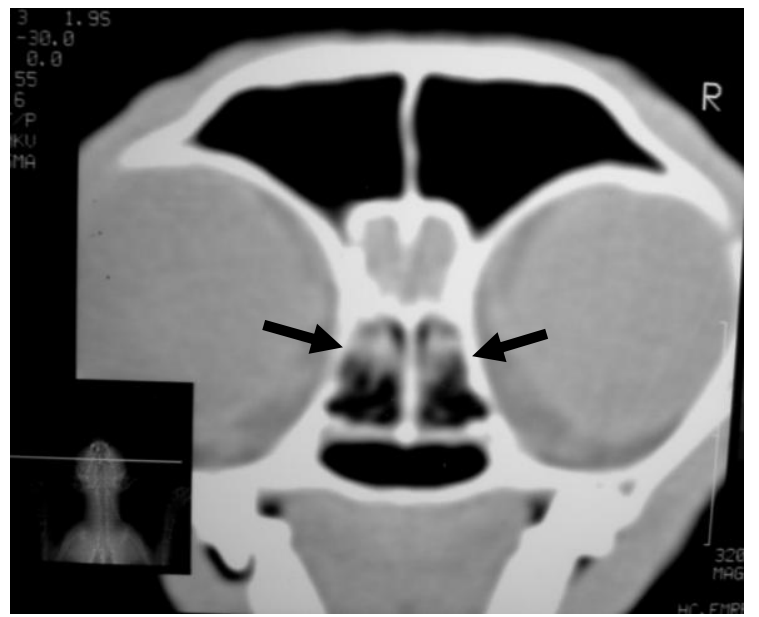

Figura 3. Imagem de tomografia computadorizada em corte transversal, utilizando janela para tecido ósseo, da cabeça de gato, sem raça definida, com sinais clínicos de doença sinonasal. Áreas radiodensas envolvendo parcialmente os seios esfenoides direito e esquerdo (seta). 
Quanto às alterações das estruturas geralmente envolvidas em quadros de doenças sinonasais, foram visibilizadas alterações tomográficas na cavidade nasal em nove dos animais. Destes, $77,7 \%$ foram modificações de opacidade (aumento), 11,1\% destruição dos turbinados nasais e $11,1 \%$ presença de massa na cavidade nasal, estendendo-se para a nasofaringe. Novamente, pode-se dizer que estes dados confirmam os já obtidos por Schoenborn et al. (2003), que concluíram que os achados tomográficos encontrados com maior frequência em gatos com doenças sinonasais são a opacificação dos seios frontais e da cavidade nasal.

Ainda, entre as alterações de estruturas extrassinonasais, em quatro gatos a tomografia detectou aumento de opacidade da cavidade timpânica. $O$ desvio de septo nasal foi identificado nas imagens tomográficas também em quatro animais, e a lise do osso lacrimal foi observada em um animal (Fig. 2).

Os grandes diferenciais do exame de TC em relação ao exame radiográfico foram a detecção de alterações nos seios esfenoides e a maior facilidade em localizar a lateralidade e o grau de envolvimento de alterações dos seios frontais. Estes resultados devem-se ao fato de a TC permitir a avaliação dessas estruturas em planos, livres de sobreposição de tecidos, o que torna possível a detecção de alterações, mesmo que discretas, ao contrário do que ocorre no exame radiográfico, em que a sobreposição de estruturas ósseas com os seios esfenoides e com os seios frontais dificulta a detecção de algumas alterações.

Durante a fase experimental, observou-se que os dois exames são de fácil realização. Em tomógrafos mais modernos, este exame pode ser realizado em menor tempo em relação ao exame radiográfico convencional (não digital) (Johnson e Wisner, 2007). Os dois métodos geram imagens utilizando radiação ionizante, o que não os diferencia quanto à segurança para paciente/pessoal. Esta relativa desvantagem dos métodos é amenizada pela necessidade de anestesia para a obtenção de imagens de qualidade da cabeça, o que favorece a contenção física do animal. É verdade que a tomografia ainda é um exame pouco disponível em relação à radiografia, por isso os exames tomográficos deste estudo não puderam ser realizados em serviços próprios para animais, pela não disponibilidade destes na região do estudo.

\section{CONCLUSÕES}

As vantagens do exame de TC em relação ao exame radiográfico, na avaliação dos seios nasais, são a detecção de alterações nos seios esfenoides e a facilidade de localização das lesões nos seios frontais, em razão da não sobreposição de estruturas nas imagens apresentadas em planos. Embora o exame radiográfico seja um método adequado para a avaliação inicial em animais com sinais de doenças sinonasais, a tomografia deve ser considerada um importante exame complementar de imagem, por fornecer informações adicionais.

\section{AGRADECIMENTOS}

Os autores agradecem ao médico veterinário autônomo José Célio F. Rolandi, à Dra. Naida Cristina Borges, do Departamento de Medicina Veterinária da Universidade Federal de Goiás, e à Dra. Angela Delete Bellucci, do Departamento de Clínica Médica da USP de Ribeirão Preto, pela ajuda na condução experimental. Durante a realização do trabalho, a autora recebeu bolsa do Conselho Nacional de Desenvolvimento Científico e Tecnológico (CNPq).

\section{REFERÊNCIAS BIBLIOGRÁFICAS}

BIRCHARD, S.J. Surgical diseases of the nasal cavity and paranasal sinuses. Semin. Vet. Med. Surg., v.10, p.77-86, 1995.

DYCE, K.M.; SACK, W.O; WENSING, C.J.G. Cabeça e parte ventral do pescoço dos carnívoros. In: DYCE, K.M.; SACK, W.O.; WENSING, C.J.G. Tratado de Anatomia Veterinária. 3.ed. Rio de Janeiro: Elsevier, 2004. p.359-384.

HAAGEN, A.J.V. Diseases of the nose and nasal sinuses. In: ETTINGER, S.J.; FELDMAN, E.C. Textbook of Veterinary Internal Medicine. Missouri: Elsevier Saunders, 2005. p.1186-1196.

HENDERSON, S.M.; BRADLEY, K.; DAY, M.J. et al. Investigation of nasal disease in the cat - a retrospective study of 77 cases. J. Feline Med. Surg., v.6, p.245-257, 2004. 
JOHNSON, E.G.; WISNER, E.R. Advances in respiratory imaging. Vet. Clin. N. Am.: Small Anim. Pract., v.37, p.879-900, 2007.

KARNIK, K.; REICHLE, J.K.; FISCHETTI, A.J. et al. Computed tomographic findings of fungal rhinitis and sinusitis in cats. Vet. Radiol. Ultrassound, v.50, p.65-68, 2009.

KEALY, J.K.; MCALLISTER, H. O crânio e a coluna vertebral. In: Radiologia e Ultrasonografia do Cão e do Gato. 3.ed. São Paulo: Manole, 2005. p.339-411.

KUEHN, N.F. Chronic rhinitis in cats. Clin. Tech. Small Anim. Pract., v.21, p.69-75, 2006.

LOSONSKY, J.M.; ABBOTT, L.C.; KURIASHKIN, I.V. Computed tomography of the normal feline nasal cavity and paranasal sinuses. Vet. Rad. Ultrassound, v.38, p.251-258, 1997.

MALINOWSKI, C. Canine and feline nasal neoplasia. Clin. Tech. Small Anim. Pract., v.21, p.89-94, 2006.

MICHIELS, L.; DAY, M.J.; SNAPS, F. et al. A retrospective study of non-specific rhinitis in 22 cats and the value of nasal cytology and histopathology. J. Fel. Med. Surg., v.5, p.279285,2003

NORRIS, A.M.; LAING, E.J. Diseases of the nose and sinuses. Vet. Clin. N. Am: Small Anim. Pract., v.15, p.865-890, 1985.

O'BRIEN, R.T.; EVANS, S.M.; WORTMAN, J.A. et al. Radiographic findings in cats with intranasal neoplasia or chronic rhinitis: 29 cases (1982-1988). J. Am. Vet. Med. Assoc., v.208, p.385-389, 1996.
PARK, R.D.; BECK, E.R.; LECOUTERUS, R.A. Comparision of computed tomography and radiography for detecting changes induced by malignant nasal neoplasia in dogs. J. Am. Vet. Med. Assoc., v.201, p.1720-1724, 1992.

POWNDER, S.; ROSE, M.; CRAWFORD, J. Radiographic techniques of the nasal cavity and sinuses. Clin. Tech. Small Anim. Pract., v.21, p.46-54, 2006.

REETZ, J.A.; MAI, W.; MURAVNICK, K.B. et al. Computed tomographic evaluation os anatomic and pathologic variations in the feline nasal septum and paranasal sinuses. Vet. Radiol. Ultrassound, v.47, p.321-327, 2006.

SCHOENBORN, W.C.; WISNER, E.R.; KASS, P.P. et al. Retrospective assessment of computed tomographic imaging of feline sinonasal disease in 62 cats. Vet. Radiol. Ultrassound, v.44, p.185195, 2003.

SCRIVANI, P.V. Sinonasal Computed Tomography in Dogs and Cats. In: IVIS Reviews in Veterinary Medicine, I.V.I.S. (Ed.). Ithaca, NY: I.V.I.S. 2009. Disponível em: <http://www.ivis.org/reviews/rev/scrivani/chapte r.asp?la=1>. Acessado em: 15 jul. 2009.

TROMBLEE, T.C.; JONES, J.C.; ETUE, A.E. et al. Association between clinical characteristics, computed tomography characteristics, and histologic diagnosis for cats with sinonasal disease. Vet. Radiol. Ultrassound, v.47, p.241248, 2006. 